\title{
Soft X-ray in-flight calibration of the ROSAT PSPC ${ }^{\star}$
}

\author{
K. Beuermann
}

\author{
Institut für Astrophysik, Friedrich-Hund-Platz 1, 37077 Göttingen, Germany \\ e-mail: beuermann@astro.physik.uni-goettingen.de
}

Received 25 January 2008 / Accepted 6 February 2008

\begin{abstract}
Aims. We present an in-flight calibration of the ROSAT PSPC using the incident spectra of the hot white dwarf HZ43 and the polar AM Her.

Methods. We derive an absolute flux calibration of the PSPC using the accurately known soft X-ray spectrum of HZ43. Corrections to the PSPC response matrix are derived from a comparison of predicted and observed PSPC spectra of HZ43, supplemented by results for AMHer.

Results. The calibration of the PSPC for photon energies $E<0.28 \mathrm{keV}$ is found to be accurate to better than $5 \%$ refuting earlier reports of a major miscalibration. Our corrections to the detector response matrices remove systematic residuals in the pulse height spectra of soft sources.
\end{abstract}

Key words. methods: data analysis - stars: individual: HZ43 - stars: individual: AM Her - X-rays: stars

\section{Introduction}

The absolute flux calibration of satellite instruments in the soft $\mathrm{X}$-ray regime is complicated by the lack of suitable flux standards. The situation is better in the keV range and much better in the optical and ultraviolet regimes, where the observed and model spectral fluxes of white dwarfs agree at the $1 \%$ level (e.g. Bohlin et al. 2001). Instruments with spectral capability in the soft X-ray regime are usually calibrated in flight by comparing the observed spectra of white dwarfs with pure hydrogen atmospheres with theoretical spectra extrapolated from optical and ultraviolet wavelengths. The absolute flux calibration of the ROSAT PSPC differs in that it is based entirely on ground calibrations (e.g. Briel et al. 1997; Schmitt \& Snowden 1990). Several authors have claimed that observations of white dwarfs indicated an actual sensitivity of the PSPC that is lower than nominal by a factor of about two (Napiwotzki et al. 1993; Jordan et al. 1994; Wolff et al. 1996, 1999). A decisive step was the establishment of HZ43 and Sirius B as soft X-ray standards by Beuermann et al. (2006). They fitted model spectra for the two white dwarfs and the neutron star RX J1856-37 ${ }^{1}$ simultaneously to the Chandra LETG+HRC spectra of the three stars obtaining the incident spectra along with a common wavelength-dependent correction to the effective area of LETG+HRC spectrometer. Based on these results, I present an absolute flux calibration of the PSPC and a correction to the detector response.

\section{Absolute calibration of the ROSAT PSPC}

Folding the incident spectrum of HZ43 through the PSPC response with the nominal effective area and detector response

\footnotetext{
* Based on observations made with the ROSAT satellite.

1 A two-blackbody model for RXJ 1856-27 and TMAP spectra for HZ43 and Sirius B. For the latter see http://astro. uni-tuebingen.de/ rauch/TMAP/UserGuide/UserGuide.html
}

matrix $^{2}$ yields PSPC spectra that closely resemble the observed ones (Beuermann et al. 2006, see their Fig. 9).

The remaining differences in flux (count rate) and spectral shape disappear if we choose values of the gain $g$ of the PSPC and the thicknesses (wavelength-dependent optical depths) $w$ of the detector window and $b$ of the boron filter that differ from nominal. Nonlinear systematic gain variations over the lifetime of the PSPC were discussed by (Prieto et al. 1996). At the low energies considered here (channels $x=8-60$ ), it suffices to consider a linear gain change that compresses or stretches the response matrix $D(x, E)$ for a given incident energy $E$ by an energy-independent, but possibly time-dependent, factor $g$. We consider $w$ or $b$ as time-independent but, of course, they may differ for PSPC C and B used before and after 25 January 1991, respectively. DRMPSPC-AO1 is the nominal detector response matrix for PSPC C and PSPC B for observations taken before 11 October 1991, when the high voltage of PSPC B was reduced, and DRMPSPC is recommended after that date.

For the observations with both PSPCs before 11 October 1991, nearly perfect agreement between the predicted and the observed PSPC spectra of HZ43 is obtained for values of the gain $g$, the window thickness $w$, and the boron filter thickness $b$ a few percent less than nominal (Table 1, lines 1 to 4). The errors quoted for these quantities include the effect of anti-correlated variations in $w$ and $b$ and the 5\% uncertainty in the absolute flux of HZ43 A (Beuermann et al. 2006, Table 3). We conclude that the absolute calibration of the PSPC is very close to nominal. In fact, both PSPCs may be

\footnotetext{
2 The nominal detector response matrices, DRMPSPC-AO1 and DRMPSPC, the effective areas of both PSPCs, and the correction vectors drived here, $c_{\mathrm{AO} 1}(x)$ and $c(x)$, are available via http://WWW . mpe. mpg.de/xray/wave/rosat/doc/calibration/index.php We use the nominal effective areas of PSPC C and PSPC B as given by effarea-pspcc and effarea-pspcb except for a minor correction that more accurately represents the structure of the carbon K-edge at $43.6 \AA$ (Freyberg, private communication).
} 

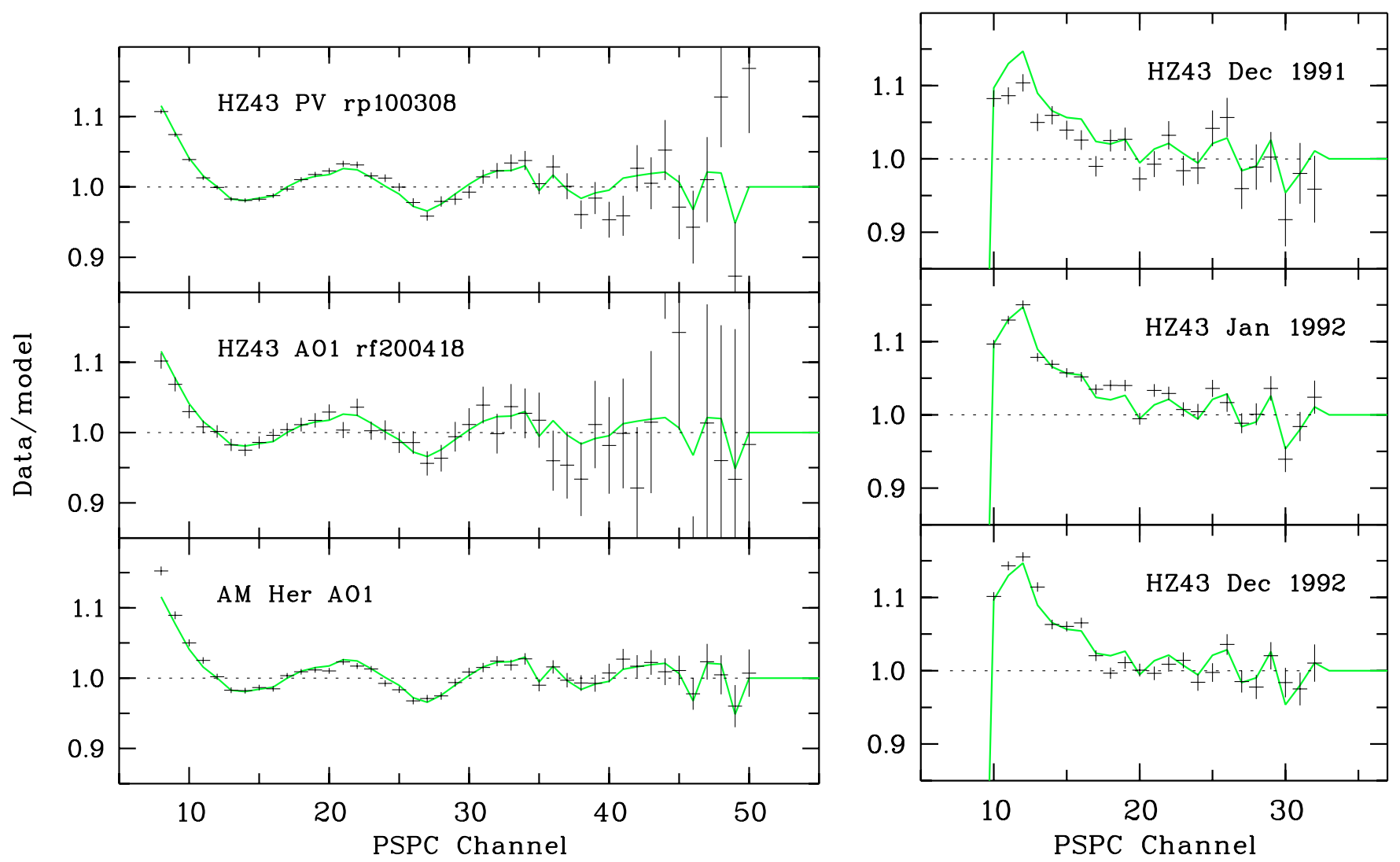

Fig. 1. Residuals of the PSPC spectra calculated for the incident spectra of the calibrator star HZ43 and the polar AM Her, derived from the Chandra LETG+HRC spectra with the LETG effective area correction of Beuermann et al. (2006) included. One PSPC channel corresponds to a nominal apparent photon energy of $10 \mathrm{eV}$. The green curves represent the weighted averages of the residuals for the PSPC observations before 11 October 1991 (left panel) and after that date (right panel). They are used to create the corrected matrices DRMPSPC-AO1c and DRMPSPCc.

Table 1. Journal of ROSAT PSPC observations of HZ43 and AM Her used in the present paper, supplemented by the fit values for $g$, $w$, and $b$ (see text). A downward arrow denotes that a parameter is kept at its previous value. The observed count rates are integrals for channel 11 to 60 .

\begin{tabular}{llrrrrcrcc}
\hline \hline Object & Identifier & Date & Exp (s) & \multicolumn{1}{c}{ Counts/s } & PSPC & open/boron & $g$ & $w$ & $b$ \\
\hline HZ43 & rp100308 & 900621 & 21520 & $66.09 \pm 0.09$ & C & open & $0.986 \pm 0.003$ & $0.988 \pm 0.015$ \\
& rf100113 & 900621 & 290 & $8.65 \pm 0.27$ & C & boron & $\downarrow$ & - \\
& rp200418 & 910619 & 262 & $64.62 \pm 0.77$ & B & open & $0.993 \pm 0.003$ & $0.963 \pm 0.015$ \\
& rf200418 & 910619 & 21625 & $8.04 \pm 0.03$ & B & boron & $\downarrow$ & & $\downarrow$ \\
& rp141808,09,12 & 911211 & 1411 & $62.23 \pm 0.32$ & B & open & $0.998 \pm 0.003$ & $\downarrow$ & $0.990 \pm 0.015$ \\
& rp141818,27,28 & 920112 & 5420 & $63.73 \pm 0.16$ & B & open & $0.998 \pm 0.005$ & $\downarrow$ & $\downarrow$ \\
& rf141822 & 920113 & 1424 & $8.12 \pm 0.12$ & B & boron & $1.005 \pm 0.005$ & $\downarrow$ & $\downarrow$ \\
& rp141916,7 & 921215 & 6901 & $62.68 \pm 0.17$ & B & open & $0.988 \pm 0.003$ & $\downarrow$ & $\downarrow$ \\
& rf141917 & 921215 & 2796 & $7.83 \pm 0.08$ & B & boron & $\downarrow$ & $\downarrow$ & $\downarrow$ \\
AM Her & r141500 & 930709 & 2663 & $7.68 \pm 0.08$ & B & boron & $0.978 \pm 0.003$ & $\downarrow$ & $\downarrow$ \\
& rp300067-0 & 910412 & 9394 & $134.27 \pm 0.13$ & B & open & $0.990 \pm 0.003$ & $\downarrow$ & $\downarrow$ \\
\hline
\end{tabular}

slightly more sensitive than nominal, as indicated by $w, b<1$. Keeping $w$ and $b$ fixed for observations with PSPC B after 11 October 1991, we obtain good fits for a relative gain that decreases slightly with time, in agreement with the results of Prieto et al. (1996). A relative gain $g \lesssim 1$ is expected from the reduced sensitivity at the center of the PSPC (Snowden et al. 2001) as the standard location for pointed observations. The central gain depression was present from the begining of the mission, but varies with time. It has a depth of about $4 \%$, but the effect is mitigated by the \pm 3 arcmin systematic oscillation of the pointing direction that moves the source back and forth across the depression with a $400 \mathrm{~s}$ period.

\section{Corrections to the detector response matrix}

The low energy resolution of the PSPC should produce smooth spectra, whereas the observed ones still contain residuals that become obvious if the statistical errors are sufficiently small. The residuals between predicted and observed spectra are shown in Fig. 1 (left panel) for the HZ43 observations before 11 October 1991. The same undulations with an amplitude of a few percent occur in both observations taken with PSPC B and PSPC C. We also show the residuals for AM Herculis computed with the Chandra LETGS observation No. 6561 with the corrected effective areas of the LETGS (Beuermann et al. 2006) and 
applying an energy-independent normalization factor of 1.03 to account for a small difference in the relative flux levels of AMHer in the Chandra and the PSPC observations. That the latter factor is so close to unity is fortuitous considering the variable character of the source. Within statistical errors, AMHer shows the same residuals as HZ43. The green curve underlying the data points for the three observations is their weighted mean. The green curve defines a correction vector $c_{\mathrm{AO} 1}(x)$ for $x \leq 50$, with $c_{\mathrm{AO} 1}(x) \equiv 1.0$ for $x>50$ and $x$ the channel number. To correct a PSPC spectrum taken prior to 11 October 1991, we either divide the observed spectrum by $c_{\mathrm{AO} 1}(x)$ or multiply all rows (constant incident photon energy) of the detector response matrix DRMPSPC-AO1 with $c_{\mathrm{AO} 1}(x)$ to obtain the corrected matrix DRMPSPC-AO1c. The observed residuals may result from non-linearities in the analog-to-digital converter and possibly from remaining uncertainties in the pulse height distributions for low-energy photons that have their centroid below the detector threshold. The residuals may, therefore, not be strictly independent of photon energy and we advise to use DRMPSPC-AO1c preferably for sources as soft as white dwarfs. This is the case for supersoft X-ray sources and for most polars observed with ROSAT.

A correction of this type has already been applied to the detector response matrix DRMPSPC valid for all observations after 11 October 1991 (Briel et al. 1997). Not surprisingly, therefore, the undulations present in the residuals of the early HZ43 observations are gone in exposures after that date (Fig. 1, right panel). The only remaining significant feature is a pile-up of counts at channel numbers below 20 peaking near threshold, which resembles the final rise below channel 13 in the left panel.
We apply this second-order correction vector $c(x)$ to the detector response matrix DRMPSPC valid after 11 October 1991 to create the corrected matrix DRMPSPCc. The caveat concerning harder sources applies here, too. In principle, a possible energy dependence of the features in both panels of Fig. 1 could be studied using sources with different, but known, incident spectra. The only source we are aware of, however, that is reasonably constant has sufficient exposure in the PSPC, and a well-exposed Chandra LETG spectrum is Capella.

Acknowledgements. We thank Vadim Burwitz for giving us the mean Chandra LETG spectrum of AM Her and Michael Freyberg for supplying the gain maps of the ROSAT PSPC. We thank Ulrich Briel, Frank Haberl, Jürgen H. M. M. Schmitt, Joachim Trümper, and other former and present colleagues at the MPE Garching for enlightening discussions on the properties of the ROSAT PSPC and its calibration.

\section{References}

Beuermann, K., Burwitz, V., \& Rauch, T. 2006, A\&A, 458, 541 Bohlin, R. C., Dickinson, M. E., \& Calzetti, D. 2001, AJ, 122, 2118

Briel, U. G., Aschenbach, B., Englhauser, J., et al. 1997, ROSAT Users's Handbook, 45

Jordan, S., Wolff, B., Koester, D., \& Napiwotzki, R. 1994, A\&A, 290, 834 Napiwotzki, R., Barstow, M. A., Fleming, T., et al. 1993, A\&A, 278, 478 Prieto, M. A., Hasinger, G., \& Snowden, S. L. 1996, A\&AS, 120, 187

Schmitt, J. H. M. M., \& Snowden, S. 1990 The physical composition of the PSPC entrance windows and the flight boron filters, Technical Note TN-ROS-MEZA10/038, MPE Garching

Snowden, S. L., Turner, T. J., \& Freyberg, M. J. 2001, ApJS, 132, 107

Wolff, B., Jordan, S., \& Koester, D. 1996, A\&A, 307, 149

Wolff, B., Koester, D., \& Lallement, R. 1999, A\&A, 346, 969 